\title{
The value of zebrafish as an integrative model in effect-directed analysis - a review
}

\author{
Carolina Di Paolo ${ }^{1 *}$, Thomas-Benjamin Seiler ${ }^{1}$, Steffen Keiter ${ }^{1,2}$, Meng Hư ${ }^{3}$, Melis Muz ${ }^{3}$, Werner Brack ${ }^{3}$ \\ and Henner Hollert ${ }^{1,4,5,6}$
}

\begin{abstract}
Bioassays play a central role in effect-directed analysis (EDA), and their selection and application have to consider rather specific aspects of this approach. Meanwhile, bioassays with zebrafish, an established model organism in different research areas, are increasingly being utilized in EDA. Aiming to contribute for the optimal application of zebrafish bioassays in EDA, this review provides a critical overview of previous EDA investigations that applied zebrafish bioassays, discusses the potential contribution of such methods for EDA and proposes strategies to improve future studies. Over the last 10 years, zebrafish bioassays have guided EDA of natural products and environmental samples. The great majority of studies performed bioassays with embryos and early larvae, which allowed small-scale and low-volume experimental setups, minimized sample use and reduced workload. Biotesting strategies applied zebrafish bioassays as either the only method guiding EDA or instead integrated into multiple bioassay approaches. Furthermore, tiered biotesting applied zebrafish methods in both screening phase as well as for further investigations. For dosing, most of the studies performed solvent exchange of extracts and fractions to dimethyl sulfoxide (DMSO) as carrier. However, high DMSO concentrations were required for the testing of complex matrix extracts, indicating that future studies might benefit from the evaluation of alternative carrier solvents or passive dosing. Surprisingly, only a few studies reported the evaluation of process blanks, indicating a need to improve and standardize methods for blank preparation and biotesting. Regarding evaluated endpoints, while acute toxicity brought limited information, the assessment of specific endpoints was of strong value for bioactivity identification. Therefore, the bioassay specificity and sensitivity to identify the investigated bioactivity are important criteria in EDA. Additionally, it might be necessary to characterize the most adequate exposure windows and assessment setups for bioactivity identification. Finally, a great advantage of zebrafish bioassays in EDA of environmental samples is the availability of mechanism- and endpoint-specific methods for the identification of important classes of contaminants. The evaluation of mechanism-specific endpoints in EDA is considered to be a promising strategy to facilitate the integration of EDA into weight-of-evidence approaches, ultimately contributing for the identification of environmental contaminants causing bioassay and ecological effects.
\end{abstract}

Keywords: Effect-directed analysis; Bioassay-guided fractionation; Zebrafish; Embryo; Larva; Bioassay; In vitro; In vivo

\section{Review} Introduction

Zebrafish is a model vertebrate organism broadly applied in biological sciences, being one of the most important organisms that is used in different research areas as genetics, developmental biology and ecotoxicology [1]. More recently, its versatility has also been recognized by

\footnotetext{
* Correspondence: carolina.dipaolo@bio5.rwth-aachen.de

'Department of Ecosystem Analysis, Institute for Environmental Research,

ABBt - Aachen Biology and Biotechnology, RWTH Aachen University,

Worringerweg 1, Aachen 52074, Germany

Full list of author information is available at the end of the article
}

chemists, which provides an opportunity to enhance interdisciplinary studies involving biology and chemistry [2] as in effect-directed analysis (EDA) [3].

\section{Bioassays in EDA}

EDA, bioassay-guided fractionation and similar approaches are testing procedures applied to identify the individual bioactive compounds contained in highly complex matrices, such as natural products and environmental samples. Bioassays play a central role in EDA since biological activity directs the chemical fractionation and analysis steps as well as the testing strategy.

\section{实}


Since fractionation of the sample is required to reduce the complexity of the original mixture, bioassays are needed to identify the active fractions and to guide further fractionation steps. Target and non-target chemical analyses are applied to select candidates and identify bioactive substances. Bioassays again play an important role in the confirmation phase, for biotesting of the pure substance identified as the bioactive compound [3-5].

Therefore, bioassay selection for EDA studies has to consider aspects that are rather specific to this application. For accurate identification of bioactive fractions, the bioassays should present high sensitivity and low internal test variability and be able to detect different chemicals that address similar endpoints or modes of action. Furthermore, due to limited sample amounts and large numbers of fractions to be tested, high-throughput low-volume bioassays are required [5].

Thus, in vitro bioassays are often selected for EDA studies; however, certain bioactivities require the organ or organism level for their proper identification, as for compounds in which metabolism plays an important role by interfering with formation or transformation of bioactive metabolites and bioaccumulation profiles [6]. These are the cases when bioassays with zebrafish earlylife stages are considered to be of great value since they combine the organism-level endpoints with advantages of the in vitro format. Furthermore, biotesting strategies integrating organism-based and in vitro bioassays are expected to cover a broad range of bioeffects and related toxicants. The resulting diagnostic power strongly supports the identification of specific toxicants in EDA case studies [7].

\section{Zebrafish model and bioassays in EDA}

The zebrafish Danio rerio exhibits characteristics that make it a very attractive research model, including small size, ease of culture, high fecundity, rapid development, external fertilization and development, and transparency of the embryo. Bioassays with zebrafish embryos and larvae have further advantages that fit very well to EDA requirements. While these tests are relevant to evaluate acute [8] and chronic [9] effects in later life stages, the experimental setup exhibits several in vitro test characteristics, including a reduced volume of sample for testing and potential for high-throughput applications. Experiments with early life stages often do not require animal test authorization, and no external feeding is needed by embryos and larvae [1].

The zebrafish success as a model organism is in great part due to the work of pioneer scientists between the late 1960s and mid-1990s, as George Streisinger, who established the first zebrafish models and performed pioneer works on its genetics and developmental biology [10-12]; Charles Kimmel's descriptions of the cellular fate map [13] and the stages of development [14] in embryos; and Christiane Nüsslein-Volhard, who performed a large-scale mutant screen to identify genes for vertebrate development control $[15,16]$. Following these ground-breaking studies, there was evident increase in the use of zebrafish in research [17], resulting in the sequencing of its genome [18], extensive information on its genetics, genomics, phenotypic and developmental biology [19], and the establishment of thousands of wildtype and transgenic zebrafish lines [20,21].

Importantly, zebrafish embryos and early larvae might be used to replace or refine experiments with adult fish, being increasingly applied in ecotoxicology to evaluate the toxicity of chemicals, plant protection products, biocides, pharmaceuticals, wastewater effluents and various aqueous environmental samples, and to assess sediment toxicity [1,22-24]. Recently, zebrafish embryo toxicity assays have been integrated in biotest batteries in environmental monitoring programmes, as the Joint Danube Survey [25] and the working group on bioassays of the NORMAN network [26]. Fish bioassays also play an important role in the implementation of the European Water Framework Directive (WFD) since they provide data for the derivation of environmental quality standards (EQS) and might represent a sensitive taxon for substances with specific modes of action [27]. Besides, biotests with fish are also included among recommended bioeffect-based tools for environmental assessment in the context of the WFD and the European Marine Strategy Framework Directive (MSFD) [7]. Consequently, current EU projects are investigating the contribution of zebrafish bioassays for water quality assessment and EDA of environmental samples, with focus on specific modes of action, mechanism-specific endpoints and adverse-outcome pathways $[28,29]$. Such initiatives are supported by the proposal that EDA contributes as an additional line of evidence in weight-of-evidence frameworks, such as the triad approach, ultimately leading to the identification of the contaminants responsible for the toxic effects observed in bioassays and the environment [30,31].

\section{Context and objectives of this review}

This review was developed in the context of the Marie Curie Initial Training Network 'EDA-EMERGE - Novel tools in effect-directed analysis for identifying and monitoring emerging toxicants on a European scale', funded by the European Commission within the Seventh Framework Programme for Research [28]. The literature review aimed to provide an overview of previous EDA investigations that applied bioassays with zebrafish, critically evaluating their objectives, methods, biotesting strategy and outcomes; discuss the potential contribution of further zebrafish bioassays for EDA; and propose strategies that might help optimizing the integration of such biotools into future EDA studies investigating environmental samples. 
In order to meet these objectives, the literature was searched using the online tools Thomson Reuters Web of Science (WoS), ScienceDirect (SD) and Google Scholar (GS). In WoS, the terms were searched by topic (searching the fields Title, Abstract, Author Keywords and Keywords Plus ${ }^{\odot}$ per record) in all databases, and in SD and GS, the terms were searched in all fields. The searches were done for publications in all years, except where indicated. The zebrafish terms used for search or filtration were a combination of 'zebrafish' or 'zebra fish' or 'Danio rerio'. The EDA and life stage search terms are detailed below.

\section{Zebrafish potential for EDA application \\ Zebrafish in EDA-relevant research areas}

The application of the zebrafish model in EDA-related research areas was verified by search in WoS for the zebrafish terms as keywords in topic/all databases, followed by classification per research area (Figure 1). The search period was limited to between 2004 and 2014 , to be in agreement with the publication years of EDA studies evaluated in this review. Outcomes are in good agreement with a recent review that applied much more sophisticated search strategy [17], indicating the usefulness of WoS for a first evaluation of research areas. Among the research areas strongly related to EDA, toxicology (8.9\%) and pharmacology (9.0\%) were each referred by circa $9 \%$ of the publications, while environmental sciences and ecology (3.0\%) and chemistry $(1.9 \%)$ were referred by a lower percentage. The prevalent research fields addressed by more than $20 \%$ of publications were mostly those that traditionally apply zebrafish, as genetics and heredity (40.6\%), biochemistry and molecular biology (33.9\%), developmental biology (30.7\%), and zoology (24.6\%)

\section{Life stages referred to by research studies}

The use of different life stages in studies with zebrafish was estimated by search for the zebrafish terms filtered by the life stage terms 'embryo*', 'larva*', 'juvenile*', 'adult"' and combinations of those. Again, the search period was limited to the publication years of reviewed EDA studies (2004 to 2014). As illustrated in Figure 2, more than half of the studies with zebrafish refer to embryos (52.6\%) and almost one fourth of these mentioned also either adults or larvae, corresponding to $4.0 \%$ and $7.4 \%$ of total publications, respectively. The occurrence of studies mentioning adults (13.4\%) and larvae (11.9\%) was also representative, while circa $1 \%$ only referred to juvenile life stages.

\section{EDA studies integrating zebrafish bioassays}

Due to the heterogeneous nomenclature found in the literature, different EDA terms as listed in Weller 2012 [4] plus the term 'fractionation' were searched in quotation

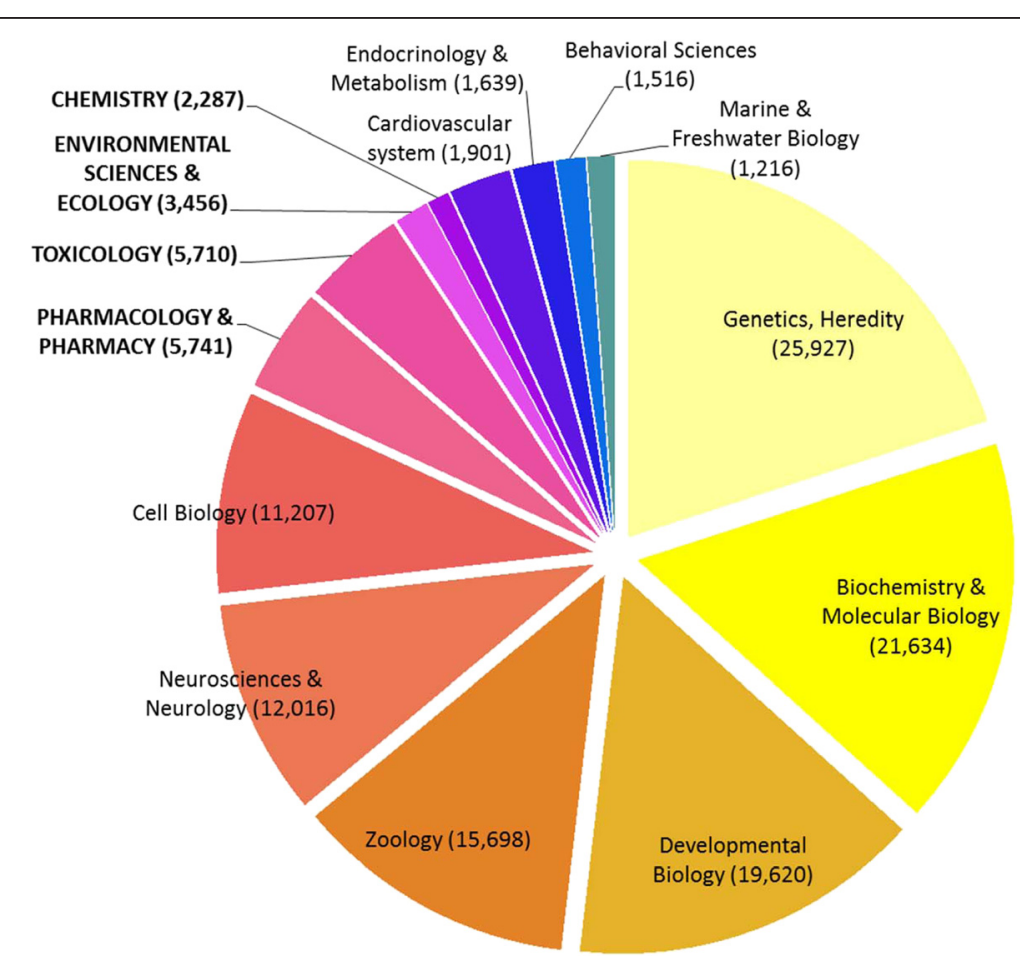

Figure 1 Records for the zebrafish terms, filtered by publication period (2004 to 2014), classified according to research area. Total number of records per period: 63,851. Search done in October 2014 (Web of Science). 


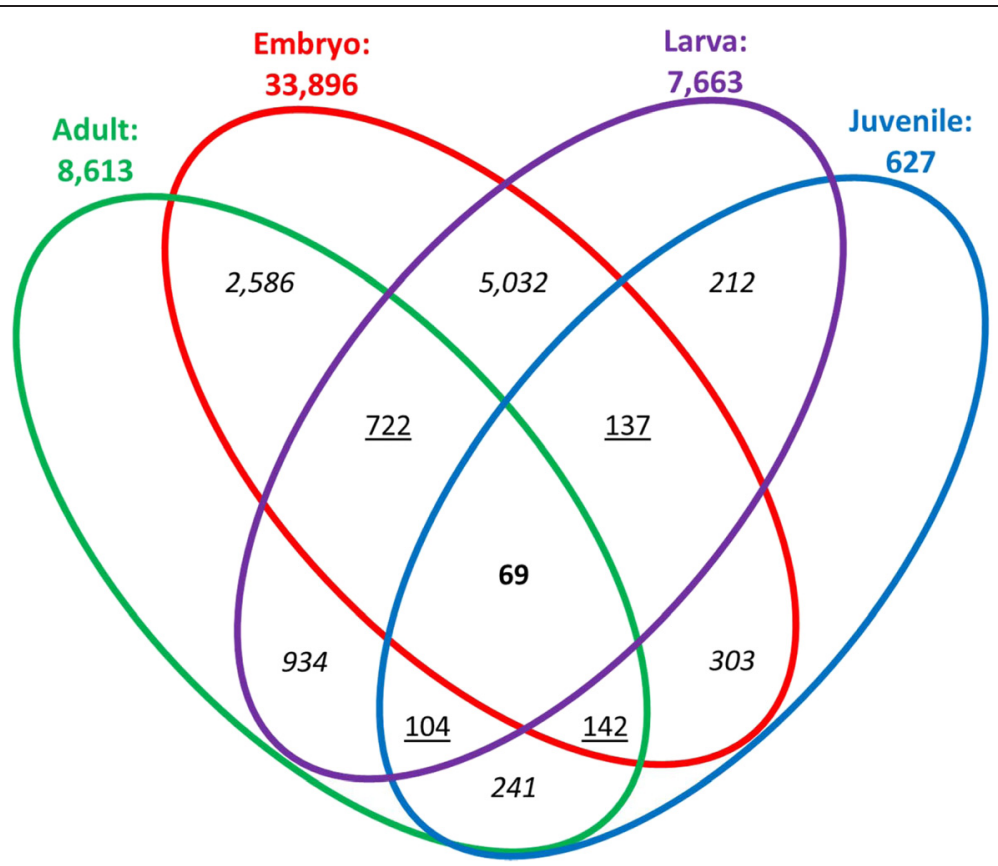

Figure $\mathbf{2}$ Records for the zebrafish and life stage terms, filtered by publication period (2004 to 2014). The life stage terms are 'embryo*' 'larva*', 'juvenile*', 'adult"* or combinations of these. Total number of records per period: 63,851. Search done in October 2014 (Web of Science).

marks, using the different search tools. After filtering by the zebrafish terms mentioned above, resulting publications were screened for confirmation of the searched content. Review papers, or studies that did not include the EDA procedure or zebrafish bioassays, were excluded. Two studies that followed procedures for toxicity identification evaluation (TIE) $[32,33]$ instead of EDA were included since the similarities between both approaches [5] make them relevant for this review. In total, 29 publications were found (Table 1), which were carefully evaluated for research area, objective, investigated matrix, bioassay endpoint and setup, biotesting strategy, and study outcomes.

\section{Research areas and investigated matrices}

Two main fields were prevalent among EDA studies using zebrafish bioassays: drug discovery from natural products and environmental toxicology (Figure 3). Natural product studies aimed to identify bioactive compounds for pharmacological applications, investigating mostly plant extracts [19,34-45] but also extracts of bacteria [46], cyanobacteria and algae [47], seaweed [48] and marine organisms [49]. Environmental toxicology studies aimed to identify the toxic compounds in various environmental samples, including marine and fluvial sediments [50-52], soil [53], cyanobacteria and algae [54,55], industrial effluent [33], rubber tyre leachates [32], oil sand process waters $[56,57]$ and river pore water [58]. Finally, fish skin extracts were investigated in a behavioural sciences study [59].

\section{Prevalent life stages and exposure setups}

EDA studies applied mostly bioassays with early embryos and larvae, following exposure to chemical extracts and fractions in multiwell-plates, often with exposure of several individuals in the same well (Table 2). Zebrafish up to 5 days post fertilization (dpf) were the life stages mostly applied, except for experiments that extended the assays up to 6 to $7 \mathrm{dpf}[34,49,53,56,57]$ or a few studies with adults $[33,47,59]$. Environmental toxicology studies for the most part performed exposure not only in 24 -well plates $(200 \mu \mathrm{L}$ to $2 \mathrm{~mL}$ per embryo or larva) but also in crystallization dishes, scintillation vials or beakers $(450 \mu \mathrm{L}$ to $5 \mathrm{~mL}$ per embryo or larva, 40 to $300 \mathrm{~mL}$ per adult), while natural product studies were performed exclusively in multiwell-plate setup $(<100$ to $250 \mu \mathrm{L}$ per embryo or larva). The exposure of several individuals in the same well or vessel was observed for most of the studies, reflecting the need to reduce workload for EDA biotesting.

\section{Biotesting strategy}

The EDA investigations guided only by zebrafish bioassays followed either a single test setup (e.g. [36,53,55-57]) or a combination of methods (e.g. $[44,52])$ to evaluate endpoints in zebrafish. Other studies applied methods with additional experimental models, mostly cell-based (e.g. $[19,45,51])$ but also bacteria $[46,50]$ and rodent [39] assays. When the application of multiple biossays aimed to evaluate distinct bioactivities, the tests were mostly performed 
Table 1 Records for the EDA terms after filtering and after confirming that studies performed EDA

\begin{tabular}{|c|c|c|c|c|}
\hline Search terms & $\begin{array}{l}\text { WoS, all databases } \\
\text { by topic }(n)\end{array}$ & $\begin{array}{l}\text { WoS, filtered by } \\
\text { zebrafish terms }(n)\end{array}$ & WoS, confirmed content $(n)$ & $\begin{array}{l}\text { Confirmed papers in } \\
\text { WoS + SD + GS (n) }\end{array}$ \\
\hline Bioassay(-)guided fractionation & 5,134 & 14 & 8 & 9 \\
\hline Effect(-)directed analysis & 189 & 14 & 3 & 5 \\
\hline Bioassay(-)guided isolation & 454 & 9 & 7 & 8 \\
\hline Bioassay(-)directed fractionation & 464 & 8 & 0 & 1 \\
\hline Toxicity(-)identification evaluation & 346 & 2 & 1 & 2 \\
\hline Bioactivity(-)screening & 92 & 3 & 2 & 2 \\
\hline Activity(-)guided fractionation & 641 & 2 & 1 & 1 \\
\hline Bioactivity(-)guided fractionation & 492 & 1 & 0 & 2 \\
\hline Bio(-)guided fractionation & 136 & 1 & 1 & 1 \\
\hline Fractionation & 232,733 & 84 & 15 & 21 \\
\hline Total $^{a}$ & & & & 29 \\
\hline
\end{tabular}

The sum of papers from WoS, ScienceDirect (SD) and Google Scholar (GS) after confirmation of content is also presented. Search done in October $2014 .{ }^{\text {a }}$ The total number differs from the sum since some studies resulted in more than one search.

in parallel. For instance, bioassay batteries evaluated the occurrence of different toxicity mechanisms [50,51] or effects on different trophic levels in the two TIE studies $[32,33]$. When instead the aim of multiple methods was to analyse different aspects of the same bioactivity or toxicity mechanism, there was the prevalence of tiered approach biotesting $[19,39,45]$. When applied in screening phase, zebrafish bioassays aimed to identify active fractions by organism-level endpoints, which were later further investigated by additional methods with zebrafish [52] or with other experimental models $[19,45]$. As an example, zebrafish bioassays were applied to screen extracts and fractions for anti-angiogenic effects, followed by further investigations on human cells and transgenic zebrafish embryos [19]. On the other hand, zebrafish bioassays applied only as secondary tests aimed mostly at the confirmation of bioeffect occurrence at the organism level [49] or to evaluate the occurrence of acute toxicity in fish $[46,47]$.

\section{Use of solvents in bioassays}

In biotesting, solvents were used for transference of samples into exposure vessels or as carriers. The first approach was applied using acetonitrile [55] or ethanol [54], including also solvent control conditions, and proceeding to solvent evaporation before adding exposure media. The use of solvents as carriers in bioassays showed the prevalence of

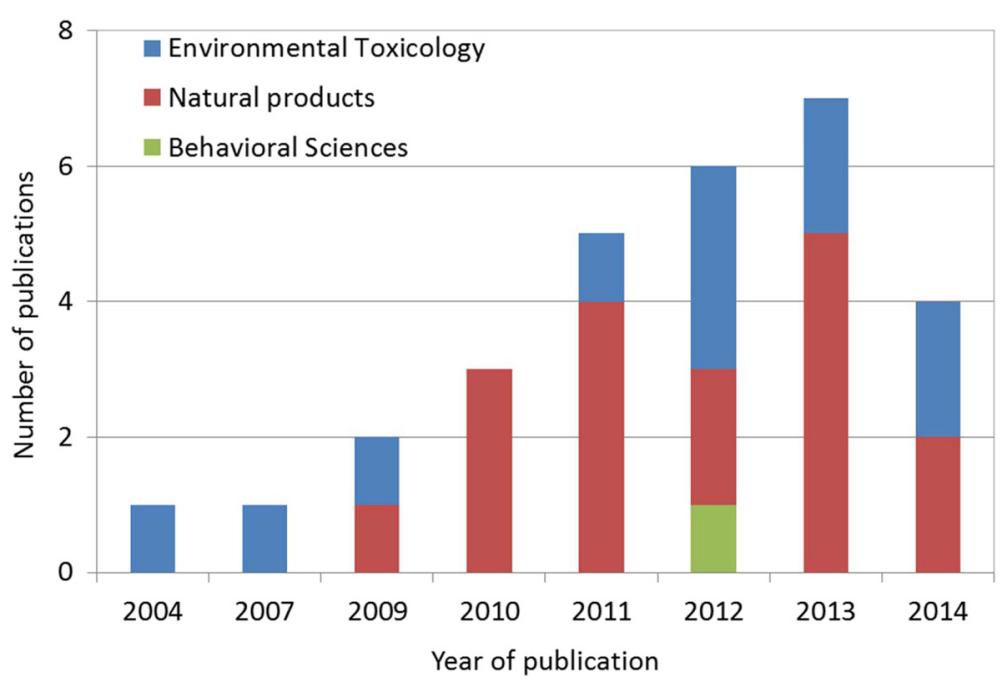

Figure 3 EDA studies applying zebrafish bioassays for the investigation of natural products and environmental samples. Number of studies that were evaluated in the literature review identified per main research area and year of publication (Web of Science, ScienceDirect and Google Scholar, October 2014). 
Table 2 Exposure setup for studies on natural products or environmental toxicology

\begin{tabular}{llll}
\hline Exposure format & $\begin{array}{l}\text { Fish per well } \\
\text { or vessel }(\boldsymbol{n})\end{array}$ & $\begin{array}{l}\text { Natural products } \\
\text { Medium volume per fish } \\
\text { and respective reference }\end{array}$ & $\begin{array}{l}\text { Environmental toxicology } \\
\text { Medium volume per fish } \\
\text { and respective reference }\end{array}$ \\
\hline 96-well plates & 1 & $100 \mu \mathrm{L}[19,30,34,39,42]$ & $100 \mu \mathrm{L}[50]$ \\
24-well plates & 4 & $<100 \mu \mathrm{L}[45]$ & - \\
& 1 & - & $2 \mathrm{~mL}[32]$ \\
& 5 & n.a. $[46]$ & $200 \mu \mathrm{LL}[51,54,55]$ \\
& 10 & 100 to $250 \mu \mathrm{L}[38,40,43]$ & - \\
12-well plates & 15 & $<100 \mu \mathrm{L}[41]$ & - \\
6-well plates & 25 & $<100 \mu \mathrm{L}[35]$ & - \\
Beakers, dishes, vials & 10 & n.a. $[48,49]$ & - \\
\hline
\end{tabular}

n.a. means information not available; ${ }^{a}$ bioassay with embryos and larvae, ${ }^{b}$ bioassays with adults.

dimethyl sulfoxide (DMSO), in concentrations ranging from $0.01 \%$ [35], $0.1 \%$ [31,45], $0.2 \%$ [26], 0.5\% [46,47], $1 \%$ [33,48-50] up to $2 \%$ [51]. In addition, ethanol was also used as a carrier by a few studies, in concentrations of $0.001 \%$ for experiments with larvae $[40,41]$ or $0.435 \%$ for experiments with zebrafish adults [42].

It is relevant to mention that the different procedures for extraction, cleanup, pre-concentration and fractionation of samples, already extensively reviewed elsewhere $[3,4,60]$, also involve the use of different solvents and chemicals. Criteria for the use of solvents in EDA studies are the following: low or lack of toxicity in the biotest, the capacity of the solvent to dissolve complex extracts and fractions and the possibility to use the solvent in chemical analysis. The latter is the precondition to make sure that the chemical mixture tested in the bioassay resembles the mixture evaluated in chemical analysis. While DMSO excellently meets the first criterion, it is less suitable for dissolving complex mixtures when compared to other possible alternatives, and it completely fails the criterion related to the use in chemical analysis. Thus, the investigation of other solvents as possible carriers for exposure in zebrafish embryo testing might help to reduce possible artefacts during solvent exchange to DMSO. The evaluation of process blanks in order to exclude artefact toxicity is crucial for successful EDA and will be discussed below.

\section{Positive/negative controls and biotesting of blanks}

Positive control conditions that were specific to the evaluated endpoints were often described. For that, there was exposure of the zebrafish to compounds known to cause specific effects such as anti-convulsant activity [39], glucose uptake [45], pro-angiogenesis [36,37], antiangiogenesis $[19,44]$, or estrogenic effects $[52,56]$. Regarding negative control conditions, most of the studies reported the testing of solvent controls in the same concentration as for the respective sample testing $[19,32,34,35,38-40,43,44,47,49-51,53,55]$. Some studies have additionally evaluated a medium only condition in addition to the solvent control $[36,37,42,54,56,57]$.

The preparation and biotesting of blanks was described only in few of the evaluated EDA studies and in the two TIE studies. In the EDA studies, there was submission of the respective solvents $[51,53,56,57]$ or of HPLC-grade water [58] through the same or part of the procedures that were applied to samples (i.e. sample preparation, extraction, fractionation). The TIE studies described blank preparation by treatment of milliQ water [32] or $0.1 \mathrm{M} \mathrm{KCl}$ solution [33] in the same way as samples for all procedures. In all these studies, the prepared blanks were evaluated in bioassays in the same way as done for samples and fractions. Another strategy was the use of a fraction that showed to be negative for the evaluated effect as a blank condition [45]. The exchange between elution solvents and DMSO was identified as a critical step since solvent traces might interfere with bioassays; therefore, blank testing was suggested to always be performed [51].

\section{Investigated endpoints and studies outcomes}

The specificity and sensitivity of bioassays and endpoints in identifying the bioactivity or adverse effects in fractions were considered to be a key issue for the relevance of zebrafish bioassays in EDA. Therefore, it is recommended to identify the critical aspects for endpoint assessment, to optimize bioassays accordingly and to demonstrate the validity of the bioassay by testing known bioactive compounds [61]. The endpoints and bioassays described in the different studies are summarized in Table 3 and discussed in the context of respective study objectives and outcomes. 
Table 3 Organism-level effects and assessed endpoints, according to the research area of studies and the investigated sample matrices

\begin{tabular}{|c|c|c|c|}
\hline Organism-level effect & Endpoint & Research area & Matrix and reference \\
\hline \multirow[t]{2}{*}{ Acute toxicity } & $\begin{array}{l}\text { Lethality and acute toxicity } \\
\text { (respiratory rate, heart rate, } \\
\text { movement) endpoints }\end{array}$ & Environmental toxicology & $\begin{array}{l}\text { Sediment extracts [50], industrial } \\
\text { effluents [33], rubber tyre leachates [32], } \\
\text { oil sand process waters [57] }\end{array}$ \\
\hline & & Natural products & $\begin{array}{l}\text { Cyanobacteria and algae extracts [47], } \\
\text { seaweed hydrolysates [48] }\end{array}$ \\
\hline \multirow[t]{2}{*}{ Acute and developmental toxicity } & Lethal and morphological endpoints & Environmental toxicology & $\begin{array}{l}\text { Sediment extracts [51], soil extracts [53], } \\
\text { microalgae extracts [54], cyanobacteria } \\
\text { extracts [55], river pore water extract [58] }\end{array}$ \\
\hline & & Natural products & Bacteria extracts [46] \\
\hline Developmental toxicity & Phenotypic endpoints & Natural products & Plant extracts [41] \\
\hline Anti- or pro-angiogenesis & $\begin{array}{l}\text { ISV formation and/or function in } \\
\text { wild-type or fli1:EGFP zebrafish }\end{array}$ & Natural products & Plant extracts [19,36-38,42-44] \\
\hline $\begin{array}{l}\text { Anti-angiogenesis and } \\
\text { anti-inflammatory }\end{array}$ & $\begin{array}{l}\text { ISV outgrowth in fli1:EGFP, leukocyte } \\
\text { migration after tail transection }\end{array}$ & Natural products & Plant extracts [40] \\
\hline Glucose uptake & $\begin{array}{l}\text { Uptake of fluorescein-tagged } \\
\text { glucose bioprobe }\end{array}$ & Natural products & Plant extracts [45] \\
\hline Lipid storage modulation & $\begin{array}{l}\text { Uptake and metabolism } \\
\text { of fluorescent fatty acid }\end{array}$ & Natural products & $\begin{array}{l}\text { Heterofibrin molecules } \\
\text { from Spongia sp. [49] }\end{array}$ \\
\hline Antioxidant effects & ROS generation, cell death & Natural products & Plant extracts [35] \\
\hline \multirow[t]{2}{*}{ Estrogenicity } & GFP induction in tg(cyp19a1b-GFP) & Environmental toxicology & Sediment extracts [52] \\
\hline & vtg1 gene expession by qPCR & Environmental toxicology & Oil sand process waters [56] \\
\hline \multirow[t]{2}{*}{ Anti-convulsant } & $\begin{array}{l}\text { Locomotor activity, electrographic } \\
\text { activity and epileptiform discharges }\end{array}$ & Natural products & Plant extracts [39] \\
\hline & $\begin{array}{l}\text { Inhibition of pentylenetetrazol-induced } \\
\text { seizure activity, WISH for brain } \\
\text { c-fos expression }\end{array}$ & Natural products & Plant extracts [34] \\
\hline Fear behaviour & $\begin{array}{l}\text { Alarm response, olfactory bulb } \\
\text { activation in Ta1:GCaMP2 }\end{array}$ & Behavioural sciences & Skin extracts [59] \\
\hline
\end{tabular}

ISV, intersegmental vessels; fli1:EGFP, transgenic zebrafish line with expression of enhanced green fluorescent protein marker in endothelial cells of vasculature; ROS, reactive oxygen species; GFP, green fluorescent protein; WISH, whole-mount in situ hybridization; Ta1:GCaMP2, transgenic zebrafish line with expression of the calcium indicator GCaM.

\section{Acute toxicity and lethality}

Bioactive sediment fractions [51] and components partially responsible for toxicity in oil sand process water fractions [57] have been identified by acute toxicity bioassays. The two TIE studies reported inconsistent acute toxicity of industrial effluents [33] and rubber tyre leachates [32]. One study investigating seaweed hydrolysates evaluated in vivo toxic potential through acute toxicity testing [48].

It may be summarized that EDA studies that focused on acute toxicity and lethality had only modest success in determining active compounds. These are unspecific responses that might occur due to exposure to very broad range of compounds; therefore, fractionation typically results in the distribution of toxicity over many different fractions. However, also other unrelated factors might have been involved, as for example, the high complexity of investigated matrices in the reviewed studies. Nevertheless, acute toxicity testing might be a powerful tool in TIE, when applied to evaluate highly contaminated sites with acute toxicity caused by compounds that are well characterized [62].

\section{Teratogenesis and developmental toxicity}

Assessment of teratogenesis and developmental effects was done in studies that identified the bioactive compounds from microalgae, cyanobacteria and plant $[41,54,55]$, river pore water [58] and developmental toxicants in soil [53]. Most studies evaluated traditionally assessed morphological endpoints, while one investigation of plant fractions focused on ectopic tail formation [41]. One study identified embryotoxicity in sediment extracts but not in respective fractions, which was attributed to losses of active compound or of synergistic effect during fractionation [50].

An aspect shared by the successful studies was the meticulous experimental characterization of the original matrices and respective fractions regarding their teratogenic effects and developmental toxicity potential. For 
instance, there was the determination of the optimal exposure period to identify a phenotype of interest that caused minimal acute toxicity [41]. Characteristic phenotypical effects were identified for specific fractions [53], also on a dose-dependent manner [54,55]. Two studies also investigated if additive or synergistic effects occurred between different fractions [55] or between aryl hydrocarbon receptor (AhR) agonists by co-exposure to a CYP1A inhibitor [58].

\section{Angiogenesis modulation}

Bioassays investigating pro- and anti-angiogenesis modulation by different bioactive plants were the most frequent studies in natural products. To this end, studies applied wild-type zebrafish $[30,42,43]$ or the transgenic fli1:EGFP [63] zebrafish line [19,36-38,40], in which the zebrafish fli1 promoter drives the expression of enhanced green fluorescent protein in blood vessels. In wild-type zebrafish, staining of the vessels was applied to facilitate scoring [30,43], while the transgenic line allowed in vivo observation of the embryonic vasculature. Selected endpoints evaluated specific cellular-morphological phenotypes, as intersegmental vessel formation. In these assays, the exposure start and duration were set to the most sensitive developmental windows related to the assessed endpoints.

All of the evaluated studies were successful in identifying at least one bioactive compound causing angiogenesis modulation, indicating that the identification of highly specific endpoints on the organism level might be a good requirement for the efficient use of zebrafish bioassays in EDA. The use of transgenic zebrafish lines is also considered to be a great asset for studies that evaluate specific morphological effects since it can facilitate endpoint observation and increase sensitivity of bioassays.

\section{Energy uptake and storage}

EDA was successful in identifying known and novel insulin-mimetic compounds in plants [45] with the contribution of zebrafish bioassays to characterize glucose uptake modulation. The study applied fluorescein-tagged glucose bioprobes and measured fluorescence by microscopy imaging and microplate reader, obtaining dose- and time-dependent responses. Another study applied a fluorescent fatty acid analogue to evaluate fatty acid storage modulation in zebrafish embryos by extracts from marine sponge [49]. In this case, the characterization of effects was done by extraction of zebrafish lipids followed by thin-layer chromatography. These studies demonstrated that the use of fluorescent bioprobes is a good tool to evaluate effects on the uptake and storage capacity of zebrafish, allowing not only for qualitative but also quantitative analysis of effects.

\section{Antioxidant effects}

Zebrafish embryos were integrated into an EDA study that identified and purified aloe vera polysaccharide with protective effects against oxidative stress [35]. Tests with zebrafish bioassays provided valuable information on organism-level responses regarding the generation of reactive oxygen species and oxidative stress-induced cell death, which were observed in a dose response manner.

\section{Estrogenicity assessment by gene expression}

Estrogenic effects were investigated in extracts and fractions of oil sand process waters by vitellogenin gene expression ( $v$ tg1) through quantitative polymerase chain reaction (qPCR) in zebrafish early larvae [56]. Estrogenicity was also assessed by the use of transgenic zebrafish embryos that exhibit green fluorescence protein expression in response to aromatase (cyp19a1b) gene induction, with confirmation of results by qPCR [52].

Gene expression analysis by qPCR showed to be a useful EDA endpoint in zebrafish embryos and larvae when background information allows the selection of specific biomarker genes for the studied effect, as for estrogenicity. The evaluation of sets of genes by qPCR is considered to be a promising strategy for endocrine disruption investigation, when following optimized experimental design regarding exposure intervals and evaluated zebrafish developmental stages [64]. The transgenic zebrafish embryos were also considered to be experimental models compatible with EDA, and their integration in future studies is expected to be facilitated by automated image analysis procedures [52].

\section{Neuroactivity and behaviour}

EDA was applied to identify anticonvulsant compounds present in plants, by co-exposure of evaluated samples with a convulsant compound, followed by the analysis of larvae total locomotor activity. Effects were assessed with video-tracking and software analysis and with electroencephalogram recording analysis [39]. Another EDA study of plant neuroactivity applied similar bioassays, in combination with larvae whole-mount in situ hybridization to assess increased brain $c$-fos gene expression as an indicator of seizure onset and brain damage [34]. Both studies identified anticonvulsant compounds, demonstrating the usefulness of the zebrafish model to identify neuroactivity in EDA. Also for neuroactivity and behaviour, the assessment of specific endpoints and setting the bioassay accordingly demonstrated to be an effective EDA biotesting approach.

The identification of neuroactive compound extracts of a mixture of red algae and cyanobacteria was investigated by a biotest battery including in vitro and organism-level methods [47]. Bioassays with zebrafish adults aimed at evaluating the neurotoxic potential of the matrix. However, 
evaluated endpoints were non-specific acute toxicity and mortality, which provided only minor contribution to the overall study outcomes.

The bioactive compounds responsible for fear behaviour response in fish were investigated by the exposure of zebrafish adults to fish skin extracts and fractions [59]. Video tracking was used to quantify alarm behaviour by measuring swimming speed and vertical position. The study identified the bioactive compound and proposed a new class of odorants that trigger alarm behaviour in fish. This study required the development of experimental setup and endpoint assessment that were specific to the evaluated behavioural alteration, confirming the importance of this step also for behavioural assessment.

\section{Summary and discussion}

Over the last 10 years, EDA studies guided by zebrafish bioassays have successfully identified bioactive or toxic compounds present in diverse biological matrices or environmental samples. Embryos and early larvae were the prevalent zebrafish life stages in these studies, with exposure being done in multiwell-plates, often with several individuals in the same well. In consequence, the sample volume for biotesting was minimized and the workload was reduced, which are important aspects in EDA. Zebrafish bioassays showed also versatility in terms of biotesting strategy, being applied alone or as a part of biotest batteries and in both screening phase as well as for further investigation of active fractions in tiered biotesting.

In spite of its limited capacity to dissolve complex matrix extracts, DMSO was the main carrier solvent applied in zebrafish bioassays. As a result, it was used in concentrations up to two orders of magnitude higher than the recommended for single compound biotesting (0.01\%) [65]. Additionally, DMSO is not suitable for chemical analysis, which restricts the characterization of samples evaluated in biotesting. Therefore, the investigation of alternative carrier solvents would be an asset for zebrafish bioassays in EDA. Passive dosing methods are also promising options, as recently done in EDA investigation of sediments through the use of silicone rods for dosing of extracts and fractions in algae bioassay [66]. In fact, a loaded polymer silicone cast has successfully been integrated in zebrafish embryo assay for dosing of polycyclic aromatic hydrocarbons [67].

The EDA procedures for sample extraction, cleanup, pre-concentration and fractionation involve the use of different solvents and chemicals. Nevertheless, while most of the studies evaluated solvent and medium control conditions, the investigation of process blanks in bioassays was reported only by a small number of studies. In addition, methods for blank preparation varied considerably between these studies. Since the biotesting of process blanks is crucial for effective EDA, there is a need to improve and standardize the procedures for their preparation and biotesting in future studies.

Most of the successful EDA studies applied specific and sensitive bioassays evaluating molecular, morphological or behavioural endpoints. Some studies optimized bioassays by identifying the most adequate exposure windows and assessment setups to maximize the specific endpoint response and minimize the interference of acute toxicity [41]. Further improvements might be achieved by advancing methods for the analysis of endpoints. For instance, the automated analysis of morphological phenotypes in transgenic or wild-type zebrafish would reduce workload and increase reliability in EDA [52]. Also, EDA of environmental samples would benefit from the analysis of bioassay results in correlation with previously characterized responses to specific classes of pollutants. That is the case of gene expression analysis of biomarker genes for specific mechanisms and modes of action. When analysed in correlation with respective gene modulation by known classes of compounds [68], biomarker gene responses might indicate the presence of certain classes of chemicals [69]. Similarly, EDA studies evaluating behavioural phenotypes to identify neuroactivity might rely in the near future on databases of behavioural profiles for different classes of compounds [70,71].

Such outcomes support the idea that EDA investigations of toxic environmental samples would benefit of the application of endpoint- and mechanism-specific methods with zebrafish. That is in fact a great advantage since mechanism-specific toxicity methods with zebrafish are broadly developed, as for AhR-mediated toxicity [72], genotoxicity [73] and neurotoxicity [74]. Furthermore, EDA guided by such zebrafish bioassays could integrate broader environmental assessment strategies, complementing effect-based approaches [7] and weightof-evidence frameworks [30]. In this way, EDA would support the identification of contaminants causing bioassay and ecological effects, and the clarification of links between ecosystem functioning and the responses at different biological levels $[30,62]$. Finally, the evaluation of toxic aquatic contaminants through EDA guided by zebrafish bioassays might improve the protection of water bodies in the context of the European WFD and MSFD [7,27]. In conclusion, endpoint- and mechanism-specific zebrafish bioassays are considered of great relevance not only for guiding EDA studies but also for integrating EDA into environmental assessment and monitoring, ultimately contributing for environmental quality improvement $[7,75]$.

\section{Conclusions}

Zebrafish bioassays have successfully guided different EDA studies; however, further method developments are still needed. Alternative dosing procedures should be investigated, and process blank preparation and biotesting 
should be standardized. Endpoint- and mechanismspecific bioassays with embryos and larvae are considered to be the most promising zebrafish biotests for future EDA of environmental samples. When integrated into broader environmental assessment strategies, EDA guided by specific zebrafish bioassays might support the identification of compounds causing bioassay and ecological effects, ultimately contributing for environmental quality improvement.

\section{Competing interests}

The authors declare that they have no competing interests.

\section{Authors' contributions}

CDP was responsible for the general design of the review and wrote the first draft of the manuscript. The other authors - TBS, SK, MH, MM, WB and HH - contributed with specific information concerning their respective expertise. All authors helped revise the draft of the manuscript. All authors read and approved the final manuscript.

\section{Acknowledgements}

The EDA-EMERGE project is supported by the EU Seventh Framework Programme (FP7-PEOPLE-2011-ITN) under the grant agreement number 290100.

\section{Author details}

'Department of Ecosystem Analysis, Institute for Environmental Research, ABBt - Aachen Biology and Biotechnology, RWTH Aachen University, Worringerweg 1, Aachen 52074, Germany. ${ }^{2}$ Man-Technology-Environment Research Centre, School of Science and Technology, Örebro University, SE-701 82 Örebro, Sweden. ${ }^{3}$ UFZ-Helmholtz Centre for Environmental Research, Permoserstrasse 15, Leipzig 04318, Germany. ${ }^{4}$ College of Resources and Environmental Science, Chongqing University, 1 Tiansheng Road, Beibei, Chongqing 400715, China. ${ }^{5}$ College of Environmental Science and Engineering and State Key Laboratory of Pollution Control and Resource Reuse, Tongji University, 1239 Siping Road, Shanghai, China. ${ }^{6}$ State Key Laboratory of Pollution Control and Resource Reuse, School of the Environment, Nanjing University, Nanjing, China.

\section{Received: 1 August 2014 Accepted: 24 February 2015}

\section{Published online: 14 March 2015}

\section{References}

1. Strähle U, Scholz S, Geisler R, Greiner P, Hollert H, Rastegar S, et al. Zebrafish embryos as an alternative to animal experiments-a commentary on the definition of the onset of protected life stages in animal welfare regulations. Reprod Toxicol. 2012;33:128-32

2. Basu S, Sachidanandan C. Zebrafish: a multifaceted tool for chemical biologists. Chem Rev. 2013;113:7952-80.

3. Brack W. Effect-directed analysis: a promising tool for the identification of organic toxicants in complex mixtures? Anal Bioanal Chem. 2003;377:397-407.

4. Weller MG. A unifying review of bioassay-guided fractionation, effectdirected analysis and related techniques. Sensors. 2012;12:9181-209.

5. Burgess RM, Ho KT, Brack W, Lamoree M. Effects-directed analysis (EDA) and toxicity identification evaluation (TIE): complementary but different approaches for diagnosing causes of environmental toxicity. Environ Toxicol Chem. 2013;32:1935-45.

6. Di Paolo C, Gandhi N, Bhavsar SP, Van den Heuvel-Greve M, Koelmans AA. Black carbon inclusive multichemical modeling of PBDE and PCB biomagnification and transformation in estuarine food webs. Environ Sci Technol. 2010;44:7548-54

7. Wernersson A S CM, Maggi C, Tusil P, Soldan P, James A, Sanchez W, et al. Technical report on aquatic effect-based monitoring tools technical report 2014-077 EU Commission. doi:102779/7260. 2014.

8. Knobel M, Busser FJ, Rico-Rico A, Kramer NI, Hermens JL, Hafner C, et al. Predicting adult fish acute lethality with the zebrafish embryo: relevance of test duration, endpoints, compound properties, and exposure concentration analysis. Environ Sci Technol. 2012;46:9690-700.

9. Volz DC, Belanger S, Embry M, Padilla S, Sanderson H, Schirmer K, et al. Adverse outcome pathways during early fish development: a conceptual framework for identification of chemical screening and prioritization strategies. Toxicol Sci. 2011;123:349-58.

10. George Streisinger. Biographical Memoirs V.68: 353-362. Washington, DC The National Academies Press; 1995.

11. Streisinger G, Walker C, Dower N, Knauber D, Singer F. Production of clones of homozygous diploid zebra fish (Brachydanio rerio). Nature. 1981;291:293-6.

12. Streisinger G, Coale F, Taggart C, Walker C, Grunwald DJ. Clonal origins of cells in the pigmented retina of the zebrafish eye. Dev Biol. 1989;131:60-9.

13. Kimmel CB, Warga RM, Schilling TF. Origin and organization of the zebrafish fate map. Development. 1990;108:581-94.

14. Kimmel CB, Ballard WW, Kimmel SR, Ullmann B, Schilling TF. Stages of embryonic development of the zebrafish. Dev Dyn. 1995;203:253-310.

15. Nüsslein-Volhard C. The zebrafish issue of development. Development 2012;139:4099-103

16. Nüsslein-Volhard C. The identification of genes controlling development in flies and fishes (Nobel Lecture). Angewandte Chemie Int Ed Eng. 1996:35:2176-87.

17. Kinth P, Mahesh G, Panwar Y. Mapping of zebrafish research: a global outlook. Zebrafish. 2013;10:510-7.

18. Howe K, Clark MD, Torroja CF, Torrance J, Berthelot C, Muffato M, et al. The zebrafish reference genome sequence and its relationship to the human genome. Nature. 2013:496:498-503.

19. Bradford Y, Conlin T, Dunn N, Fashena D, Frazer K, Howe DG, et al. ZFIN: enhancements and updates to the zebrafish model organism database. Nucleic Acids Res. 2010;39(1):D822-9.

20. Zebrafish International Resource Center - ZIRC [http://www.zebrafish.org]

21. EZRC - European Zebrafish Resource Center [http://www.ezrc.kit.edu]

22. Braunbeck T, Boettcher M, Hollert H, Kosmehl T, Lammer E, Leist E, et al. Towards an alternative for the acute fish $\mathrm{LC}(50)$ test in chemical assessment: the fish embryo toxicity test goes multi-species - an update. Altex. 2005;22:87-102.

23. Hallare A, Seiler T-B, Hollert $H$. The versatile, changing, and advancing roles of fish in sediment toxicity assessment-a review. J Soils Sediments. 2011;11:141-73.

24. EURL-ECVAM. Recommendation on the zebrafish embryo acute toxicity test method (ZFET) for acute aquatic toxicity testing. Report EUR 26710. 35p. In: Recommendation on the zebrafish embryo acute toxicity test method (ZFET) for acute aquatic toxicity testing. Report EUR 26710. 35p. 2014.

25. Joint Danube Survey [http://www.danubesurvey.org]

26. NORMAN Network [http://www.norman-network.net]

27. European Commission. Technical guidance for deriving environmental quality standard. Guidance document n²7, technical report 2011-055, European Communities, 203 p. In: Technical guidance for deriving environmental quality standard. Guidance document $n^{\circ} 27$, technical report 2011-055, European Communities, 203 p. 2011

28. Brack W, Govender S, Schulze T, Krauss M, Hu M, Muz M, et al. EDA-EMERGE: an FP7 initial training network to equip the next generation of young scientists with the skills to address the complexity of environmental contamination with emerging pollutants. Environ Sci Eur. 2013;25:18.

29. Brack W, Altenburger R, Schüürmann G, Krauss M, López Herráez D, van Gils J, et al. The SOLUTIONS project: challenges and responses for present and future emerging pollutants in land and water resources management. Sci Total Environ. 2014:503-504:22-31.

30. Hecker M, Hollert H. Effect-directed analysis (EDA) in aquatic ecotoxicology: state of the art and future challenges. Environ Sci Pollut Res. 2009;16:607-13.

31. Chapman PM, Hollert H. Should the sediment quality triad become a tetrad, a pentad, or possibly even a hexad? J Soils Sediments. 2006;6:4-8.

32. Wik A, Nilsson E, Källqvist T, Tobiesen A, Dave G. Toxicity assessment of sequential leachates of tire powder using a battery of toxicity tests and toxicity identification evaluations. Chemosphere. 2009:77:922-7.

33. Fang $Y-X$, Ying G-G, Zhang L-J, Zhao J-L, Su H-C, Yang B, et al. Use of TIE techniques to characterize industrial effluents in the Pearl River Delta region. Ecotoxicol Environ Saf. 2012;76:143-52

34. Buenafe OE, Orellana-Paucar A, Maes J, Huang H, Ying X, De Borggraeve W, et al. Tanshinone IIA exhibits anticonvulsant activity in zebrafish and mouse seizure models. ACS Chem Neurosci. 2013;4:1479-87.

35. Kang MC, Kim SY, Kim YT, Kim EA, Lee SH, Ko SC, et al. In vitro and in vivo antioxidant activities of polysaccharide purified from aloe vera (Aloe barbadensis) gel. Carbohydr Polym. 2014;99:365-71.

36. Liu C-L, Cheng L, Kwok H-F, Ko C-H, Lau T-W, Koon C-M, et al. Bioassayguided isolation of norviburtinal from the root of Rehmannia glutinosa, exhibited angiogenesis effect in zebrafish embryo model. J Ethnopharmacol. 2011;137:1323-7. 
37. Liu C-L, Kwok H-F, Cheng L, Ko C-H, Wong C-W, Wai Fong Ho T, et al. Molecular mechanisms of angiogenesis effect of active sub-fraction from root of Rehmannia glutinosa by zebrafish sprout angiogenesis-guided fractionation. J Ethnopharmacol. 2014;151:565-75.

38. Crawford AD, Liekens S, Kamuhabwa AR, Maes J, Munck S, Busson R, et al, Zebrafish bioassay-guided natural product discovery: isolation of angiogenesis inhibitors from East African medicinal plants. PLoS One. 2011;6:e14694.

39. Orellana-Paucar AM, Serruys A-SK, Afrikanova T, Maes J, De Borggraeve W, Alen J, et al. Anticonvulsant activity of bisabolene sesquiterpenoids of Curcuma longa in zebrafish and mouse seizure models. Epilepsy Behav. 2012;24:14-22.

40. Bohni N, Cordero-Maldonado ML, Maes J, Siverio-Mota D, Marcourt L, Munck $\mathrm{S}$, et al. Integration of Microfractionation, qNMR and zebrafish screening for the in vivo bioassay-guided isolation and quantitative bioactivity analysis of natural products. PLoS One. 2013:8:e64006.

41. Gebruers E, Cordero-Maldonado ML, Gray Al, Clements C, Harvey AL, Edrada-Ebel $\mathrm{R}$, et al. A phenotypic screen in zebrafish identifies a novel small-molecule inducer of ectopic tail formation suggestive of alterations in non-canonical Wnt/PCP signaling. PLoS One. 2013;8:e83293.

42. Krill D, Madden J, Huncik K, Moeller PD. Induced thyme product prevents VEGF-induced migration in human umbilical vein endothelial cells. Biochem Biophys Res Commun. 2010;403:275-81.

43. Han L, Yuan Y, Zhao L, He Q, Li Y, Chen X, et al. Tracking antiangiogenic components from Glycyrrhiza uralensis Fisch. based on zebrafish assays using high-speed countercurrent chromatography. J Sep Sci. 2012;35:1167-72.

44. He MF, Liu L, Ge W, Shaw PC, Jiang R, Wu LW, et al. Antiangiogenic activity of Tripterygium wilfordii and its terpenoids. J Ethnopharmacol. 2009;121:61-8.

45. Lee J, Jung D-W, Kim W-H, Um J-I, Yim S-H, Oh WK, et al. Development of a highly visual, simple, and rapid test for the discovery of novel insulin mimetics in living vertebrates. ACS Chem Biol. 2013:8:1803-14.

46. Dash S, Nogata Y, Zhou X, Zhang Y, Xu Y, Guo X, et al. Poly-ethers from Winogradskyella poriferorum: antifouling potential, time-course study of production and natural abundance. Bioresour Technol. 2011;102:7532-7.

47. Suyama TL, Cao Z, Murray TF, Gerwick WH. Ichthyotoxic brominated diphenyl ethers from a mixed assemblage of a red alga and cyanobacterium: structure clarification and biological properties. Toxicon. 2010;55:204-10.

48. Fitzgerald C, Gallagher E, O'Connor P, Prieto J, Mora-Soler L, Grealy M, et al. Development of a seaweed derived platelet activating factor acetylhydrolase (PAF-AH) inhibitory hydrolysate, synthesis of inhibitory peptides and assessment of their toxicity using the Zebrafish larvae assay. Peptides. 2013;50:119-24

49. Rae J, Fontaine F, Salim AA, Lo HP, Capon RJ, Parton RG, et al. Highthroughput screening of Australian marine organism extracts for bioactive molecules affecting the cellular storage of neutral lipids. PLoS One. 2011;6:e22868

50. Higley E, Grund S, Jones P, Schulze T, Seiler T, Lubcke-von VU, et al. Endocrine disrupting, mutagenic, and teratogenic effects of upper Danube River sediments using effect-directed analysis. Environ Toxicol Chem/SETAC. 2012;31(5):1053-62. Epub 2012 Mar 2023.

51. Kammann U, Biselli S, Hühnerfuss $H$, Reineke $N$, Theobald N, Vobach M, et al. Genotoxic and teratogenic potential of marine sediment extracts investigated with comet assay and zebrafish test. Environ Pollut. 2004:132:279-87.

52. Fetter E, Krauss M, Brion F, Kah O, Scholz S, Brack W. Effect-directed analysis for estrogenic compounds in a fluvial sediment sample using transgenic cyp19a1b-GFP zebrafish embryos. Aquat Toxicol. 2014;154:221-9.

53. Legler J, van Velzen $\mathrm{M}$, Cenijn $\mathrm{PH}$, Houtman CJ, Lamoree $\mathrm{MH}$, Wegener JW Effect-directed analysis of municipal landfill soil reveals novel developmental toxicants in the zebrafish Danio rerio. Environ Sci Technol. 2011:45:8552-8.

54. Berry JP, Gantar M, Gibbs PDL, Schmale MC. The zebrafish (Danio rerio) embryo as a model system for identification and characterization of developmental toxins from marine and freshwater microalgae. Comp Biochem PhysiolPart C Toxicol Pharmacol. 2007;145:61-72.

55. Jaja-Chimedza A, Gantar M, Gibbs PDL, Schmale MC, Berry JP. Polymethoxy1-alkenes from aphanizomenon ovalisporum inhibit vertebrate development in the zebrafish (Danio rerio) embryo model. Mar Drugs. 2012;10:2322-36.

56. Reinardy HC, Scarlett AG, Henry TB, West CE, Hewitt LM, Frank RA, et al. Aromatic naphthenic acids in oil sands process-affected water, resolved by GCxGC-MS, only weakly induce the gene for vitellogenin production in zebrafish (Danio rerio) larvae. Environ Sci Technol. 2013:47:6614-20.
57. Scarlett AG, Reinardy HC, Henry TB, West CE, Frank RA, Hewitt LM, et al. Acute toxicity of aromatic and non-aromatic fractions of naphthenic acids extracted from oil sands process-affected water to larval zebrafish. Chemosphere. 2013:93:415-20.

58. Fang M, Getzinger GJ, Cooper EM, Clark BW, Garner LVT, Giulio RTD, et al. Effect-directed analysis of Elizabeth river pore water: developmental toxicity in zebrafish (Danio rerio). Environ Toxicol Chem. 2014:n/a-n/a.

59. Mathuru Ajay S, Kibat C, Cheong Wei F, Shui G, Wenk Markus R, Friedrich Rainer W, et al. Chondroitin fragments are odorants that trigger fear behavior in fish. Curr Biol. 2012;22:538-44.

60. Brack W, editor. Effect-directed analysis of complex environmental contamination, Handbook of environmental chemistry series 15. Berlin, Heidelberg: Springer; 2011. p. 345.

61. Cordero-Maldonado ML, Siverio-Mota D, Vicet-Muro L, Wilches-Arizábala IM, Esquerra CV, de Witte PAM, et al. Optimization and pharmacological validation of a leukocyte migration assay in zebrafish larvae for the rapid In Vivo bioactivity analysis of anti-inflammatory secondary metabolites. PLoS One. 2013;8:e75404

62. Connon RE, Geist J, Werner I. Effect-based tools for monitoring and predicting the ecotoxicological effects of chemicals in the aquatic environment. Sensors. 2012;12:12741-71.

63. Lawson ND, Weinstein BM. In vivo imaging of embryonic vascular development using transgenic zebrafish. Dev Biol. 2002;248:307-18.

64. Schiller V, Wichmann A, Kriehuber R, Muth-Kohne E, Giesy JP, Hecker M, et al. Studying the effects of genistein on gene expression of fish embryos as an alternative testing approach for endocrine disruption. Comp Biochem Physiol Toxicol Pharmacol. 2013;157:41-53.

65. OECD. Test No. 236: Fish Embryo Acute Toxicity (FET) Test. Paris: OECD Publishing; 2013

66. Bandow N, Altenburger R, Streck G, Brack W. Effect-directed analysis of contaminated sediments with partition-based dosing using green algae cell multiplication inhibition. Environ Sci Technol. 2009;43:7343-9.

67. Seiler TB, Best N, Fernqvist MM, Hercht H, Smith KE, Braunbeck T, et al. PAH toxicity at aqueous solubility in the fish embryo test with Danio rerio using passive dosing. Chemosphere. 2014;112:77-84.

68. Kosmehl T, Otte JC, Yang L, Legradi J, Bluhm K, Zinsmeister C, et al. A combined DNA-microarray and mechanism-specific toxicity approach with zebrafish embryos to investigate the pollution of river sediments. Reprod Toxicol. 2012;33:245-53

69. Keiter S, Peddinghaus S, Feiler U, von der Goltz B, Hafner C, Ho N, et al DanTox - a novel joint research project using zebrafish (Danio rerio) to identify specific toxicity and molecular modes of action of sediment-bound pollutants. J Soils Sediments. 2010;10:714-7.

70. Ali S, Champagne DL, Richardson MK. Behavioral profiling of zebrafish embryos exposed to a panel of 60 water-soluble compounds. Behav Brain Res. 2012;228:272-83.

71. Kokel D, Bryan J, Laggner C, White R, Cheung CY, Mateus R, et al. Rapid behavior-based identification of neuroactive small molecules in the zebrafish. Nat Chem Biol. 2010;6:231-7.

72. Schiwy S, Braunig J, Alert H, Hollert H, Keiter SH. A novel contact assay for testing aryl hydrocarbon receptor (AhR)-mediated toxicity of chemicals and whole sediments in zebrafish (Danio rerio) embryos. Environ Sci Pollut Res Int. 2014.

73. Kosmehl T, Hallare AV, Reifferscheid G, Manz W, Braunbeck T, Hollert H. A novel contact assay for testing genotoxicity of chemicals and whole sediments in zebrafish embryos. Environ Toxicol Chem/SETAC. 2006:25:2097-106

74. de Esch C, Slieker R, Wolterbeek A, Woutersen R, de Groot D. Zebrafish as potential model for developmental neurotoxicity testing: a mini review. Neurotoxicol Teratol. 2012;34:545-53.

75. Brack W, Klamer HJ, Lopez De Alda M, Barcelo D. Effect-directed analysis of key toxicants in European river basins a review. Environ Sci Pollut Res Int. 2007;14:30-8. 